\title{
Fresnel-transform's quantum correspondence and quantum optical ABCD Law
}

\author{
Fan Hong-Yi and Hu Li-Yun* \\ Department of Physics, Shanghai Jiao Tong University, Shanghai, 200030, P.R. China \\ ${ }^{*}$ Corresponding author: hlyun@sjtu.edu.cn.
}

February 6, 2020

\begin{abstract}
Corresponding to Fresnel transform there exists a unitary operator in quantum optics theory, which could be named Fresnel operator (FO). We show that the multiplication rule of FO naturally leads to the quantum optical ABCD law. The canonical operator methods as mapping of ray-transfer ABCD matrix is explicitly shown by FO's normally ordered expansion through the coherent state representation and the technique of integration within an ordered product of operators. We show that time evolution of the damping oscillator embodies the quantum optical ABCD law.
\end{abstract}

PACS: 42.50.-p, 42.25.Bs, 03.65.Ud

In classical optics, ray-transfer matrices, $M=\left(\begin{array}{cc}A & B \\ C & D\end{array}\right), A D-B C=1$, have been used to describe the geometrical formation of images by a centered lens system. For an optical ray (a centered spherical wavefront) passing through optical instruments there is a famous law, named $\mathrm{ABCD}$ law, governing the relation between input ray $\left(r_{1}, \alpha_{1}\right)$ and output ray $\left(r_{2}, \alpha_{2}\right)$, i.e.

$$
\left(\begin{array}{l}
r_{2} \\
\alpha_{2}
\end{array}\right)=M\left(\begin{array}{l}
r_{1} \\
\alpha_{1}
\end{array}\right),
$$


where $r_{1}$ is the ray height from the optical axis, and $\alpha_{1}$ is named the optical direction-cosine, $r_{1} / \alpha_{1} \equiv R_{1}$ specifies the ray's wavefront shape. Eq. (1) implies

$$
R_{2} \equiv \frac{r_{2}}{\alpha_{2}}=\frac{A R_{1}+B}{C R_{1}+D}
$$

This law is the core of matrix optics, since it tells us how the curvature of a centered spherical wavefront changes from one reference plane to the next. Besides, the multiplication rule of matrix optics implies that if the ray-transfer matrices of the $n$ optical components are $M_{1}, M_{2}, M_{3}, \cdots, M_{n}$, respectively, then the whole system is determined by a matrix $M=M_{1} M_{2} M_{3} \cdots M_{n}$.

One of the remarkable things of modern optics is the case with which geometrical ray-transfer methods, constituting the matrix optics, can be adapted to describe the generation and propagation of Laser beams. In 1965 Kogelnik [1] pointed out that propagation of Gaussian beam also obeys $\mathrm{ABCD}$ law via optical diffraction integration, i.e. the input light field $f\left(x_{1}\right)$ and output light field $g\left(x_{2}\right)$ are related to each other by so-called Fresnel integration [2] $g\left(x_{2}\right)=$ $\int_{-\infty}^{\infty} \mathcal{K}\left(A, B, C ; x_{2}, x_{1}\right) f\left(x_{1}\right) d x_{1}$, where

$$
\mathcal{K}\left(A, B, C ; x_{2}, x_{1}\right)=\frac{1}{\sqrt{2 \pi i B}} \exp \left[\frac{i}{2 B}\left(A x_{1}^{2}-2 x_{2} x_{1}+D x_{2}^{2}\right)\right] .
$$

The ABCD law for Gaussian beam passing through an optical system is 3 .

$$
q_{2}=\frac{A q_{1}+B}{C q_{1}+D}
$$

where $q_{1}\left(q_{2}\right)$ represents the complex curvature of the input (output) Gaussian beam, Eq. (4) has the similar form as Eq. (2). An interesting and important question naturally arises: Does ABCD law also exhibit in quantum optics? Since classical Fresnel transform should have its quantum optical counterpart (we may name it Fresnel operator (FO)), this question also challenges us if there exist corresponding multiplication rule of FO which corresponds to $M=M_{1} M_{2} M_{3} \cdots M_{n}$ ?

In the following we derive $\mathrm{ABCD}$ law in quantum optics through introducing the appropriate $\mathrm{FO}$ and exhibiting its multiplication rule. We begin with mapping the symplectic transform in complex 
$z$ space $z \rightarrow s z-r z^{*}$ onto operator $U(r, s)$ by virtue of the coherent state representation [4, 5]

$$
\begin{aligned}
U(r, s) & =\sqrt{s} \int \frac{d^{2} z}{\pi}\left|s z-r z^{*}\right\rangle\langle z| \\
& \equiv \sqrt{s} \int \frac{d^{2} z}{\pi}\left|\left(\begin{array}{cc}
s & -r \\
-r^{*} & s^{*}
\end{array}\right)\left(\begin{array}{c}
z \\
z^{*}
\end{array}\right)\right\rangle\left\langle\left(\begin{array}{c}
z \\
z^{*}
\end{array}\right)\right|,
\end{aligned}
$$

where $s$ and $r$ are complex and satisfy the unimodularity condition $s s^{*}-r r^{*}=1,|z\rangle=\exp \left(-\frac{1}{2}|z|^{2}+z a^{\dagger}\right)|0\rangle \equiv$ $\left|\left(\begin{array}{c}z \\ z^{*}\end{array}\right)\right\rangle, a^{\dagger}$ is the Bose creation operator, $\left[a, a^{\dagger}\right]=1$. Using the normal ordering of vacuum projector $|0\rangle\langle 0|=: \exp \left(-a^{\dagger} a\right)$ : and the technique of integration within an ordered product (IWOP) of operators [6, 7] we are able to calculate the integral in Eq.(5) and derive its normally ordered form

$$
U(r, s)=\sqrt{\frac{1}{s^{*}}}: \exp \left[-\frac{r}{2 s^{*}} a^{\dagger 2}+\left(\frac{1}{s^{*}}-1\right) a^{\dagger} a+\frac{r^{*}}{2 s^{*}} a^{2}\right]:,
$$

which we name the FO. Using the overlap $\left\langle z \mid z^{\prime}\right\rangle=\exp \left[-\frac{1}{2}\left(|z|^{2}+\left|z^{\prime}\right|^{2}\right)+z^{*} z^{\prime}\right]$ and the IWOP technique we can obtain multiplication rule of $U(r, s)$,

$$
U(r, s) U\left(r^{\prime}, s^{\prime}\right)=\sqrt{s s^{\prime}} \int \frac{d^{2} z d^{2} z^{\prime}}{\pi^{2}}\left|s z-r z^{*}\right\rangle\left\langle z \mid s^{\prime} z^{\prime}-r^{\prime} z^{\prime *}\right\rangle\left\langle z^{\prime}\right|=U\left(r^{\prime \prime}, s^{\prime \prime}\right),
$$

where

$$
\left(\begin{array}{cc}
s & -r \\
-r^{*} & s^{*}
\end{array}\right)\left(\begin{array}{cc}
s^{\prime} & -r^{\prime} \\
-r^{*} & s^{\prime *}
\end{array}\right)=\left(\begin{array}{cc}
s^{\prime \prime} & -r^{\prime \prime} \\
-r^{* \prime \prime} & s^{* \prime \prime}
\end{array}\right),\left|s^{\prime \prime}\right|^{2}-\left|r^{\prime \prime}\right|^{2}=1,
$$

or $s^{\prime \prime}=s s^{\prime}+r r^{*}, r^{\prime \prime}=r s^{* *}+r^{\prime} s$. To see the ABCD law more explicitly, we make the identification $z=\frac{1}{\sqrt{2}}(x+i p)$

$$
|z\rangle=\left|\left(\begin{array}{c}
x \\
p
\end{array}\right)\right\rangle=\exp [i(p X-x P)]|0\rangle, X=\frac{a+a^{\dagger}}{\sqrt{2}}, P=\frac{a-a^{\dagger}}{\sqrt{2} i},
$$

and

$$
s=\frac{1}{2}[A+D-i(B-C)], r=-\frac{1}{2}[A-D+i(B+C)],
$$

where the unimodularity $s s^{*}-r r^{*}=1$ becomes $A D-B C=1$, which guarantees the classical 
Poisson bracket invariant. Accordingly, Eq.(15) is re-expressed as

$$
\begin{aligned}
U(r, s) & =\frac{\sqrt{A+D-i(B-C)}}{\sqrt{2}} \int \frac{d x d p}{2 \pi}\left|\left(\begin{array}{cc}
A & B \\
C & D
\end{array}\right)\left(\begin{array}{l}
x \\
p
\end{array}\right)\right\rangle\left\langle\left(\begin{array}{l}
x \\
p
\end{array}\right)\right| \\
& \equiv F(A, B, C) .
\end{aligned}
$$

and Eq. (6) becomes

$$
\begin{aligned}
& F(A, B, C)=\sqrt{\frac{2}{A+D+i(B-C)}}: \exp \left\{\frac{A-D+i(B+C)}{2[A+D+i(B-C)]} a^{\dagger 2}\right. \\
& \left.+\left[\frac{2}{A+D+i(B-C)}-1\right] a^{\dagger} a-\frac{A-D-i(B+C)}{2[A+D+i(B-C)]} a^{2}\right\}: .
\end{aligned}
$$
then follows from Eqs.(17) and (88) the multiplication rule for $F$ is $F\left(A^{\prime}, B^{\prime}, C^{\prime}, D^{\prime}\right) F(A, B, C, D)=$ $F\left(A^{\prime \prime}, B^{\prime \prime}, C^{\prime \prime}, D^{\prime \prime}\right)$, where

$$
\left(\begin{array}{cc}
A^{\prime \prime} & B^{\prime \prime} \\
C^{\prime \prime} & D^{\prime \prime}
\end{array}\right)=\left(\begin{array}{cc}
A^{\prime} & B^{\prime} \\
C^{\prime} & D^{\prime}
\end{array}\right)\left(\begin{array}{cc}
A & B \\
C & D
\end{array}\right)=\left(\begin{array}{cc}
A^{\prime} A+B^{\prime} C & A^{\prime} B+B^{\prime} D \\
C^{\prime} A+D^{\prime} C & C^{\prime} B+D^{\prime} D
\end{array}\right) .
$$

To prove that $F$ is just the Fresnel operator responsible for classical Fresnel transform in Eq.(3), we derive $F$ 's canonical operator form. For this aim, we notice that when $B=0, A=1, C \rightarrow C / A$, $D=1$, Eq. (12) becomes

$$
F(1,0, C / A)=\sqrt{\frac{2}{2-i C / A}}: \exp \left[\frac{i C / A}{2-i C / A} \frac{\left(a^{\dagger 2}+2 a^{\dagger} a+a^{2}\right)}{2}\right]:=\exp \left(\frac{i C}{2 A} X^{2}\right),
$$

which is named quadratic phase operator $[8$, where in the last step we have used the operator identity [6]

$$
e^{\lambda X^{2}}=\frac{1}{\sqrt{1-\lambda}}: \exp \left[\frac{\lambda}{1-\lambda} X^{2}\right]:
$$

When $C=0, A=1, B \rightarrow B / A, D=1$, Eq.(12) reduces to

$$
F(1, B / A, 0)=\sqrt{\frac{2}{2+i B / A}}: \exp \left[\frac{i B / A}{2+i B / A} \frac{\left(a^{\dagger 2}-2 a^{\dagger} a+a^{2}\right)}{2}\right]:=\exp \left(-\frac{i B}{2 A} P^{2}\right),
$$

which is named Fresnel propagator in free space, where we have used the following operator identity

$$
e^{\lambda P^{2}}=\frac{1}{\sqrt{1-\lambda}}: \exp \left[\frac{\lambda}{1-\lambda} P^{2}\right]:
$$


When the decomposing is

$$
\left(\begin{array}{ll}
A & B \\
C & D
\end{array}\right)=\left(\begin{array}{cc}
1 & 0 \\
C / A & 1
\end{array}\right)\left(\begin{array}{cc}
A & 0 \\
0 & A^{-1}
\end{array}\right)\left(\begin{array}{cc}
1 & B / A \\
0 & 1
\end{array}\right),
$$

we immediately see $F(A, B, C)$ having its canonical operator $(X, P)$ representation

$$
\begin{aligned}
F(A, B, C) & =F(1,0, C / A) F(A, 0,0) F(1, B / A, 0) \\
& =\exp \left(\frac{i C}{2 A} X^{2}\right) \exp \left(-\frac{i}{2}(X P+P X) \ln A\right) \exp \left(-\frac{i B}{2 A} P^{2}\right),
\end{aligned}
$$

here $F(A, 0,0)$ is the squeezing operator [9, 10, 11]

$$
\begin{aligned}
F(A, 0,0) & =\operatorname{sech}^{1 / 2} \sigma: \exp \left[\frac{1}{2} a^{\dagger 2} \tanh \sigma+(\operatorname{sech} \sigma-1) a^{\dagger} a-\frac{1}{2} a^{2} \tanh \sigma\right]: \\
& =\exp \left(-\frac{i}{2}(X P+P X) \ln A\right)
\end{aligned}
$$

where $A \equiv e^{\sigma}, \frac{A-A^{-1}}{A+A^{-1}}=\tanh \sigma$. Using the canonical operator form of $F$ we can deduce its matrix element in the coordinate states $|x\rangle$ (its conjugate state is $|p\rangle$ )

$$
\begin{aligned}
\left\langle x^{\prime}|F(A, B, C)| x\right\rangle & =e^{\frac{i C}{2 A} x^{\prime 2}}\left\langle x^{\prime}\right| \exp \left[-\frac{i}{2}(X P+P X) \ln A\right] \int_{-\infty}^{\infty} d p e^{-\frac{i B}{2 A} p^{2}}\langle p \mid x\rangle \\
& =e^{\frac{i C}{2 A} x^{\prime 2}}\left\langle x^{\prime}\left|\int_{-\infty}^{\infty} \frac{d p}{\sqrt{A}} e^{-\frac{i B}{2 A} p^{2}}\right| p / A\right\rangle\langle p \mid x\rangle \\
& =\frac{1}{2 \pi} e^{\frac{i C}{2 A} x^{\prime 2}} \int_{-\infty}^{\infty} \frac{d p}{\sqrt{A}} e^{-\frac{i B}{2 A} p^{2}+i p\left(x^{\prime} / A-x\right)} \\
& =\frac{1}{\sqrt{2 \pi i B}} \exp \left[\frac{i}{2 B}\left(A x^{2}-2 x^{\prime} x+D x^{\prime 2}\right)\right]
\end{aligned}
$$

which is just the kernel of Fresnel transform in Eq.(3), this is why we name $F(A, B, C)$ the FO (though it can be named a $\mathrm{SU}(1,1)$ generalized squeezing operator either). The above discussions demonstrate how to transit classical Fresnel transform to FO (and its decomposition of canonical operators) through the coherent state and the IWOP technique.

Now we directly use the FO to derive ABCD law in quantum optics. From Eq.(12) we see that the FO generates

$$
F(A, B, C)|0\rangle=\sqrt{\frac{2}{A+i B-i(C+i D)}} \exp \left\{\frac{A-D+i(B+C)}{2[A+D+i(B-C)]} a^{\dagger 2}\right\}|0\rangle,
$$


if we identify

$$
\frac{A-D+i(B+C)}{A+D+i(B-C)}=\frac{q_{1}-i}{q_{1}+i}
$$

then

$$
F(A, B, C)|0\rangle=\sqrt{-\frac{2 /(C+i D)}{q_{1}+i}} \exp \left[\frac{q_{1}-i}{2\left(q_{1}+i\right)} a^{\dagger 2}\right]|0\rangle,
$$

The solution of Eq.(23) is

$$
q_{1} \equiv-\frac{A+i B}{C+i D}
$$

Let $F(A, B, C)|0\rangle$ expressed by (24) be an input state for an optical system which is charactristic by parameters $A^{\prime}, B^{\prime}, C^{\prime}, D^{\prime}$, then the quantum optical $A B C D$ law states that the output state is

$$
F\left(A^{\prime}, B^{\prime}, C^{\prime}\right) F(A, B, C)|0\rangle=\sqrt{\frac{-2 /\left(C^{\prime \prime}+i D^{\prime \prime}\right)}{q_{2}+i}} \exp \left[\frac{q_{2}-i}{2\left(q_{2}+i\right)} a^{\dagger 2}\right]|0\rangle,
$$

which has the similar form as Eq.(24), where $\left(C^{\prime \prime}, D^{\prime \prime}\right)$ is determined by Eq.(13), and

$$
\bar{q}_{2}=\frac{A^{\prime} \bar{q}_{1}+B^{\prime}}{C^{\prime} \bar{q}_{1}+D^{\prime}}, \quad \bar{q}_{i} \equiv-q_{i}, \quad(i=1,2)
$$

which resembles Eq.(4).

Proof:

According to the multiplication rule of two FOs and Eqs.(12)-(13) we have

$$
\begin{aligned}
& F\left(A^{\prime}, B^{\prime}, C^{\prime}\right) F(A, B, C)|0\rangle \\
& =\sqrt{\frac{2}{A^{\prime \prime}+D^{\prime \prime}+i\left(B^{\prime \prime}-C^{\prime \prime}\right)}} \exp \left\{\frac{A^{\prime \prime}-D^{\prime \prime}+i\left(B^{\prime \prime}+C^{\prime \prime}\right)}{2\left[A^{\prime \prime}+D^{\prime \prime}+i\left(B^{\prime \prime}-C^{\prime \prime}\right)\right]} a^{\dagger 2}\right\}|0\rangle \\
& =\sqrt{\frac{2}{A^{\prime}(A+i B)+B^{\prime}(C+i D)-i C^{\prime}(A+i B)-i D^{\prime}(C+i D)}} \\
& \times \exp \left\{\frac{A^{\prime}(A+i B)+B^{\prime}(C+i D)+i C^{\prime}(A+i B)+i D^{\prime}(C+i D)}{2\left[A^{\prime}(A+i B)+B^{\prime}(C+i D)-i C^{\prime}(A+i B)-i D^{\prime}(C+i D)\right]} a^{\dagger 2}\right\}|0\rangle \\
& =\sqrt{\frac{-2 /(C+i D)}{A^{\prime} q_{1}-B^{\prime}-i\left(C^{\prime} q_{1}-D^{\prime}\right)}} \exp \left\{\frac{A^{\prime} q_{1}-B^{\prime}+i\left(C^{\prime} q_{1}-D^{\prime}\right)}{2\left[A^{\prime} q_{1}-B^{\prime}-i\left(C^{\prime} q_{1}-D^{\prime}\right)\right]} a^{\dagger 2}\right\}|0\rangle .
\end{aligned}
$$

Using Eq.(25) we see $\frac{2 /(C+i D)}{C^{\prime} q_{1}-D^{\prime}}=-2 /\left(C^{\prime \prime}+i D^{\prime \prime}\right)$, together using Eq.(27) we can reach Eq.(26), thus the law is proved. Using Eq. (25) we can re-express Eq.(27) as

$$
q_{2}=-\frac{A^{\prime}(A+i B)+B^{\prime}(C+i D)}{C^{\prime}(A+i B)+D^{\prime}(C+i D)}=-\frac{A^{\prime \prime}+i B^{\prime \prime}}{C^{\prime \prime}+i D^{\prime \prime}}
$$


which is in consistent to Eq.(25). Eqs. (24)-(29) are therefore self-consistent.

As an application of quantum optical ABCD law, we apply it to tackle the time-evolution of a time-dependent harmonic oscillator whose Hamiltonian is

$$
H=\frac{1}{2} e^{-2 \gamma t} P^{2}+\frac{1}{2} \omega_{0}^{2} e^{2 \gamma t} X^{2}, \quad \hbar=1
$$

where we have set the initial mass $m_{0}=1, \gamma$ denotes damping. Using $u(t)=e^{\frac{i \gamma}{2} X^{2}} e^{-\frac{i \gamma t}{2}(X P+P X)}$ to perform the transformation

$$
\begin{aligned}
& u(t) X u^{-1}(t)=e^{-\gamma t} X, \\
& u(t) P u^{-1}(t)=e^{\gamma t} P-\gamma e^{\gamma t} X,
\end{aligned}
$$

then $i \frac{\partial|\psi(t)\rangle}{\partial t}=H|\psi(t)\rangle$ leads to $i \frac{\partial|\phi\rangle}{\partial t}=\mathcal{H}|\phi\rangle,|\phi\rangle=u(t)|\psi(t)\rangle$,

$$
H \rightarrow \mathcal{H}=u(t) H u^{-1}(t)-i u(t) \frac{\partial u^{-1}(t)}{\partial t}=\frac{1}{2} P^{2}+\frac{1}{2} \omega^{2} X^{2}, .
$$

where $\omega^{2}=\omega_{0}^{2}-\gamma^{2} \cdot \mathcal{H}$ does not contain $t$ explicitly. The dynamic evolution of a mass-varying harmonic oscillator from the Fock state $|0\rangle$ at initial time to a squeezed state at time $t$ is

$$
|\psi(t)\rangle_{0}=u^{-1}(t)|0\rangle=e^{\frac{i \gamma t}{2}(X P+P X)} e^{-\frac{i \gamma}{2} X^{2}}|0\rangle,
$$

if we let $A=D=1, B=0, C=-\gamma$; and $A^{\prime}=e^{-\gamma t}, D^{\prime}=e^{\gamma t}, B^{\prime}=C^{\prime}=0$, then $q_{1}=\frac{1}{\gamma-i}$, $q_{2}=\frac{e^{-2 \gamma t}}{\gamma-i}$, according to Eq.(26) we directly obtain

$$
u^{-1}(t)|0\rangle=\sqrt{\frac{2 e^{-\gamma t}}{e^{-2 \gamma t}+i \gamma+1}} \exp \left[\frac{e^{-2 \gamma t}-1-i \gamma}{2\left(e^{-2 \gamma t}+1+i \gamma\right)} a^{\dagger 2}\right]|0\rangle,
$$

so the time evolution of the damping oscillator embodies the quantum optical ABCD law.

In summary, the operator methods as mapping of ray-transfer ABCD matrix has been explicitly shown through FO's normally ordered form and the coherent state representation. The multiplication rule of FO naturally leads to the quantum optical version of ABCD law in classical optics. Therefore, the ABCD law not only exists in classical optics, but also exhibits in quantum optics, this is a new resemblance between the two fields. The fractional Hankel transform studied in the context of quantum optics is shown in Refs. [12, 13].

This work was supported by the National Natural Science Foundation of China under grant 10475056. 


\section{References}

[1] Kogelnik H, 1965 Applied Opt. 41562

[2] J. W. Goodman, Introduction to Fourier Optics, McGraw-Hill, New York, 1972

[3] Chen B X and Sun B H, 2003 Chin. Phys. Lett. 20 1254;

Gerrard A and Burch J M, Introduction to Matrix method in optics, Wiley, London, 1975

[4] Glauber R J, 1963 Phys. Rev. 130 2529; 1963 Phys. Rev. 1312766.

[5] Klauder J R, Sudarshan E C G, Fundamentals of Quantum Optic W. A. Benjamin, New York, 1968.

[6] Fan H Y, Lu H L and Fan Y, 2006 Ann. of Phys. 321 480; Fan H Y, 2003 J. Opt. B: Quantum E) Semiclass. Opt. 5 R147

[7] Wünsche A, 1999 J. Opt. B: Quantum \&3 Semiclass. Opt. 1 R11

[8] Nazarathy M, Shamir J, 1982 J. Opt. Soc. Am 72356

[9] Fan H Y and FAN Y, 2002 Chin. Phys. Lett. 19 159;

Fan H Y and Liu N L, 2001 Chin. Phys. Lett. 18322

[10] Loudon R and Knight P L, 1987 J. Mod. Opt. 34 709; Mandel L and Wolf E, Optical Coherence and Quantum Optics, Cambridge, 1995

[11] Review Article: Dodonov V V, 2002 J. Opt. B: Quantum 8 Semiclass. Opt. 4 R1

[12] Fan H Y and Lu H L, 2003 Opt. Lett. 28680.

[13] Fan H Y and Lu H L, 2003. Opt. Lett. 282177 